\title{
Steering System of Cylindrical Elastic Crawler Robot
}

\author{
Jun-ya Nagase $^{* a)}$ Non-member, Fumika Fukunaga* Non-member \\ Kan Ishida $^{* *} \quad$ Non-member, Norihiko Saga ${ }^{* * *} \quad$ Member
}

(Manuscript received Jan. 15, 2018, revised April 16, 2018)

\begin{abstract}
Many different types of pipeline installations exist, such as pipelines used in chemical plants, water pipes and gas pipes. All must be examined and maintained to prevent severe accidents through a program of continual pipe inspection and repair. Herein, we have developed a cylindrical crawler robot for pipe inspection. We propose a simple crawler robot steering principle along with verification tests. The prototype robot with the proposed steering mechanism was able to travel through all T-branch paths.
\end{abstract}

Keywords: in-pipe robot, cylindrical crawler robot, steering mechanism, slider

\section{Introduction}

Pipe installations are used throughout industry and society, as pipelines in chemical plants, but also as water pipes and gas pipes for residences. Severe accidents must be prevented for all such installations through continual pipe inspection and repair. Therefore, the use of autonomous pipe-inspection robots is anticipated. Such robots can be inserted automatically into a pipe by the driving force of actuators.

We have developed a cylindrical elastic crawler robot ${ }^{(1)}$ for pipe-inspection with high running performance in vertical pipes, and in corners and uneven narrow pipe areas. This extremely simple structure contrasts against conventional tracked-crawler robots, which have complicated structures and many actuators ${ }^{(2)(3)}$. However, devices of our earlier design cannot move in an arbitrary direction at branch joints such as T corners. Herein, we newly propose a steering mechanism for cylindrical elastic crawler robots. This report describes the structure and principle of the proposed steering mechanism and its verification testing.

\section{Cylindrical Elastic Crawler Robot}

Figure 1 portrays the structure of the cylindrical elastic crawler robot. This mechanism is a cylindrical crawler comprising a single geared motor, silicone rubber crawler belts, a single worm, and a resin frame. Six crawler belts are spaced at equal intervals around the longitudinal axis of the cylindrical frame. Each crawler belt is wound around the frame: both ends are connected, producing a loop. Additionally, teeth having a rake angle corresponding to the lead angle of the worm tooth are formed on the crawler belt outer surface. The geared motor is mounted as stationary inside and coaxially to the frame. The worm, which is attached to the motor shaft, is positioned to engage with each of the six crawler belts.

Regarding the driving principle, first, the motor fixed to the frame creates rotation via the gear. After the rotating worm

a) Correspondence to: Jun-ya Nagase. E-mail: nagase@ @ins. ryukoku.ac.jp

* Ryukoku University

1-5, Yokotani, Seta Oe-cho, Otsu, Shiga 520-2194, Japan

** Okayama University

3-1-1, Tsushima-naka, Kita-ku, Okayama 700-8530, Japan

*** Kwansei Gakuin University

2-1, Gakuen, Sanda, Hyogo 669-1337, Japan

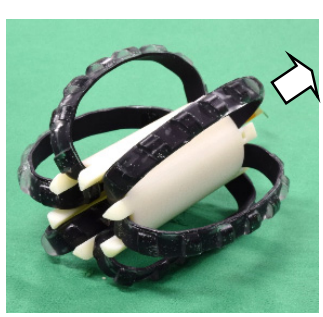

(a) Prototype

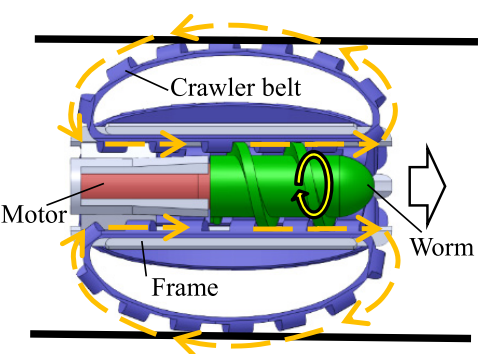

(b) Section
Fig. 1. Cylindrical elastic crawler robot ${ }^{(1)}$

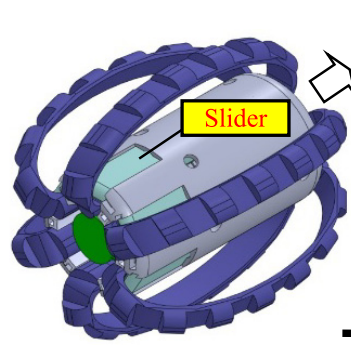

(a) Overview

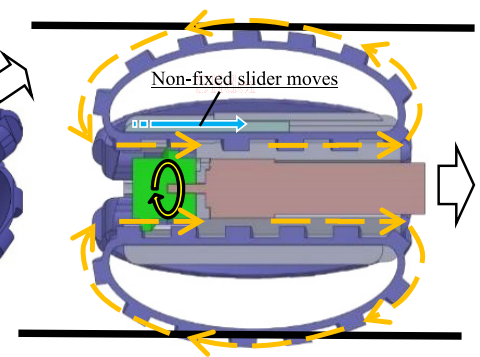

(b) Section
Fig. 2. Cylindrical elastic crawler robot with steering mechanism engages with all six crawler belts, the crawler belt rotates around the frame. Then the crawler robot moves forward by the force transmitted from the crawler belt to the frame. Moreover, the inverse rotation of the motor produces backward movement. The prototyped crawler presented in Fig. 1 has $92 \mathrm{~mm}$ total length and $0.13 \mathrm{~kg}$ mass. The outer diameter of the robot with maximum deformation of the crawler belt is $46 \mathrm{~mm}$. The outer diameter when not stressed by external forces is $90 \mathrm{~mm}$.

\section{Steering Mechanism}

3.1 Structure Figure 2 portrays the structure of the proposed steering mechanism. There are sliders in the frame of the robot. Each slider is arranged between the rear end of the crawler belt and the frame, respectively, as presented in Fig. 2. They can be fixed or non-fixed individually. When the slider is fixed with the frame, the driving force $F_{t}$ of the crawler belt is transmitted to the frame. Thereby, it gives a propulsive force to the crawler robot. By contrast, when the slider is not fixed with the frame, the slider slides against the 


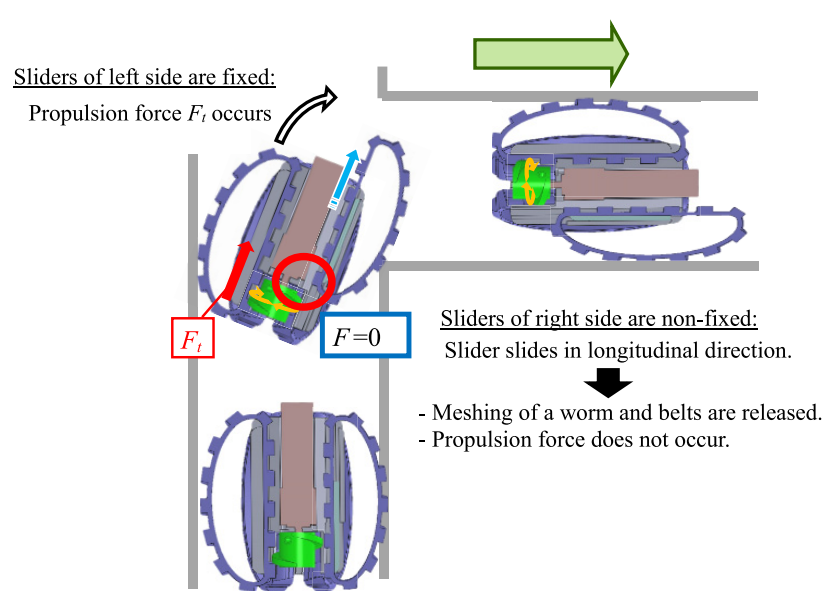

Fig. 3. Steering principle of the proposed steering mechanism

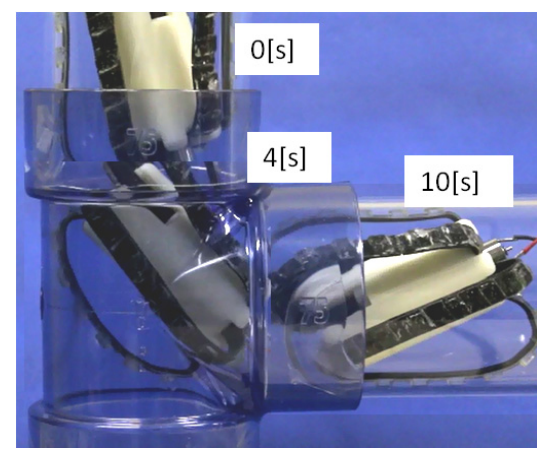

Fig. 4. Experiment result

frame in a longitudinal direction by the driving of the crawler belt. The crawler robot has no propulsive force.

3.2 Steering Principle The crawler robot with the steering mechanism can change its moving direction using the fixed slider and the non-fixed slider at branch joints. For example, it is assumed that three fixed sliders are on the left side of the robot, and that three non-fixed sliders are on the right side, as depicted in Fig. 3. In this case, the propulsion force occurs only on the left side of the robot. Furthermore, three non-fixed sliders on the right side of the robot move in a longitudinal direction until these three crawler belts on the right side are not engaged with a worm. Consequently, the robot turns right. If three fixed sliders are on right side of the robot, and non-fixed three sliders are on the left side of them, then the robot turns left as described above.

3.3 Verification Test A verification test of the proposed steering mechanism was conducted using a prototype. The prototype has $92 \mathrm{~mm}$ total length and $0.17 \mathrm{~kg}$ mass. The outer diameter of the prototype when the belts maximally deformed is $49 \mathrm{~mm}$. Its outer diameter is $93 \mathrm{~mm}$ when not stressed by external forces. The crawler belts are made of silicone rubber. The frame, the worm, and sliders are made of ABS resin. A $10.6 \mathrm{~W}$ DC geared motor was used. The experiment examined whether the prototype can change its moving direction at branches of acrylic T-shaped pipes. The pipe inner diameter is $77 \mathrm{~mm}$. The experiment results are portrayed in Fig. 4 and Table 1. Consequently, we observed that the prototype was able to change its moving direction using the proposed steering mechanism. Additionally, it was able to run all of the routes shown in Table 1.
Table 1. Running routes and its experiment result

\begin{tabular}{|c|c|c|c|c|c|c|}
\hline & & & & & \multirow{2}{*}{$\stackrel{5}{\longleftrightarrow}$} & \multirow{2}{*}{$\sqrt[6]{\square}$} \\
\hline Routes & 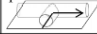 & $\ll$ & $\Longrightarrow$ & ( & & \\
\hline Propriety & 0 & 0 & 0 & 0 & 0 & $\mathrm{O}$ \\
\hline \multicolumn{7}{|c|}{ (b) Vertical T-branch 1} \\
\hline \multirow{3}{*}{ Routes } & 1 斥 & 2 & 3 & 4 & 5 & $6 \sqrt{\overline{1}}$ \\
\hline & $\Longrightarrow$ & $\square$ & $\longleftrightarrow$ & & $\longrightarrow$ & $\square$ \\
\hline & $\triangle$ & $\downarrow$ & 1 & $\downarrow$ & $\lambda \longrightarrow$ & $\Lambda$ \\
\hline Propriety & $\mathrm{O}$ & $\mathrm{O}$ & $\mathrm{O}$ & $\mathrm{O}$ & $\mathrm{O}$ & $\mathrm{O}$ \\
\hline \multicolumn{7}{|c|}{ (c) Vertical T-branch 2} \\
\hline \multirow{3}{*}{ Routes } & $\bar{\uparrow}$ & 2 & \begin{tabular}{|ll}
3 & $T$
\end{tabular} & 4 & 5 & \\
\hline & $\square$ & 7 & $\longleftrightarrow$ & r & $\longleftrightarrow$ & \\
\hline & $\checkmark$ & $\downarrow$ & $\checkmark u$ & $\downarrow$ & 11 & 11 \\
\hline Propriety & 0 & $\mathrm{O}$ & 0 & 0 & $\mathrm{O}$ & $\mathrm{O}$ \\
\hline \multicolumn{7}{|c|}{ (d) Vertical T-branch 3} \\
\hline \multirow{2}{*}{ Routes } & $\vec{\square} \square 0$ & 40 & $3 \rightleftharpoons 0$ & ${ }^{4} \longrightarrow$ & $\sqrt[5]{\square}$ & $6 \longleftarrow$ \\
\hline & 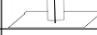 & 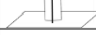 & $\downarrow$ & 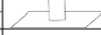 & $v$ & 54 \\
\hline Propriety & 0 & 0 & 0 & 0 & 0 & 0 \\
\hline \multicolumn{7}{|c|}{ Vertical T-branch 4} \\
\hline Routes & \begin{tabular}{l|l|} 
& \\
&
\end{tabular} & ${ }^{2} \leftarrow$ & $\begin{array}{ll}3 & \uparrow \\
\end{array}$ & & $5 \longdiv { \uparrow }$ & \\
\hline Propriety & $\mathrm{O}$ & O & $\mathrm{O}$ & O & $\mathrm{O}$ & $\mathrm{O}$ \\
\hline
\end{tabular}

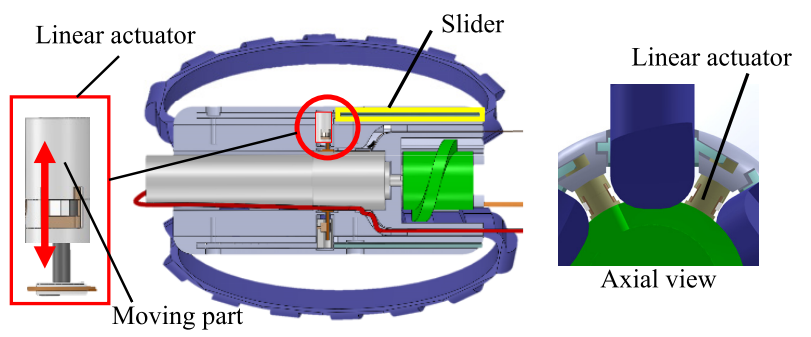

Fig. 5. Switch mechanism using linear actuators

\section{Conclusion}

As described herein, we proposed a novel steering mechanism for the cylindrical elastic crawler robot, and conducted verification tests to assess it. During the experiment, the prototyped robot was able to go pass through all routes at branches of T-shaped corners.

As future work, we expect to develop switch mechanisms to control the slider, as presented in Fig. 5. This mechanism has small linear actuators that can switch the slider between fixed and non-fixed configurations. The moving part of the linear actuator can move upward and downward. When the moving part is up, the slider is fixed. When it is down, the slider is not fixed. We will also develop a restoration mechanism with springs between each slider and each frame. The springs can restore the slider to its original position. We would like to produce a crawler robot for which the moving direction can be controlled in an arbitrary direction using these mechanisms.

Acknowledgment

This research was supported by JKA and promotion funds from KEIRIN RACE.

\section{References}

( 1 ) J. Nagase and F. Fukunaga: "Development of a novel crawler mechanism for pipe inspection", Proc. 42nd Annual Conference of the IEEE Industrial Electronics Society, IECON 2016, pp.5873-5878 (2016)

( 2 ) J. Park, D. Hyun, W. Cho, T. Kim, and H. Yang: "Normal-Force Control for an In-Pipe Robot According to the Inclination of Pipelines", IEEE Tans. Industrial Electronics, Vol.91, No.10, pp.7003-3005 (2003)

( 3 ) J. Kim, G. Sharma, and S. Iyengar: "FAMPER: A Fully Autonomous Mobile Robot for Pipeline Exploration", Proc. 2010 IEEE /ICIT International Conference on Industrial Technology, pp.517-523 (2010) 